\title{
The Application of BP Neural Network in the Thrust Hydraulic System for Shield Tunneling Machine
}

\author{
Geqiang $\mathrm{Li}^{1,3,{ }^{*} \text {, Yinting Ding }}{ }^{1,3}$, Kui Chen ${ }^{2,3}$, Weifeng Han $^{2,3}$ and Bingjing Guo ${ }^{1}$ \\ ${ }^{1}$ Henan University of Science and Technology, Jianxi Borough 471003, Luoyang China \\ ${ }^{2}$ State Key Laboratory of Shield Machine and Boring Technology, Zhengzhou 450001, Henan, China \\ ${ }^{3}$ Collaborative Innovation Center of Machinery Equipment Advanced Manufacturing of Henan Province, Henan University of \\ Science and Technology, Luoyang 471003, Henan, China
}

\begin{abstract}
This paper studies the problem of the precision of thrust speed and pressure compound control under the uncertain external load condition for shield tunneling machine. An controller about thrust speed and pressure is designed based on the traditional PID control algorithm combined with BP neural networks. By using AMEsim and Simulink software, the physical model and controller of the thrust system are established and joint simulation is conducted. The thrust system of shield machine model is simulated under the condition of different load and flow, which proves the stability and robustness of the controller with BP neural network algorithm.
\end{abstract}

Keywords-shield machine; thrust hydraulic system; BP neural network; simulation

\section{INTRODUCTION}

As the subway tunnel and underground space construction in full swing, shield tunneling machine is increasingly applied to the project construction popularly. The thrust system of shield mainly completes the thrust, attitude adjustment, direction of adjustment and so on. Its control performance is important to the precision of the driving route and the useful life of the cutter head. , Many factors cause serious impact on the control performance of thrust system and the quality of the tunnel construction, such as the complexity of the soil environment and time-varying load, etc [1]. Therefore, the control of thrust system for shield tunneling machine has become the problem of shield construction.

Many scholars at home and abroad studied the control strategy of thrust hydraulic system. Gong Guo-fang et al. applied the fuzzy PID control strategy to study the response characteristics of shield thrust speed; Liu Guo-bin, et al. put forward neural network control strategy and showed the effectiveness with the mathematical model of the thrust speed[2].Hu Shi, et al. designed the single neuron PID controller of thrust system and realized the self-adaptive control of thrust speed. Zhou Rulin, et al. designed interval synchronization controller of the thrust system, in which combined the expert system with PID control strategy. $\mathrm{Hu}$ Guo-liang et al. adopted master-slave synchronous PID controller, and verified the performance on the test bench. The present achievements of research mostly focused on studying the adaptability of thrust speed of shield, but few on studying the mutual influence between thrust pressure and speed.

Given this, the PID control strategy based on BP neural networks is applied to the thrust speed and pressure compound control in this paper. Under the conditions of variable load and flow, the control characteristics of pressure and speed of thrust hydraulic system are analyzed.

\section{ShIEld Hydraulic THRUST System PrinCIPLE}

The hydraulic cylinders of thrust system adopt zone control. The thrust speed of cylinders from each zone is adjusted to meet the requirements of turning, curved propelling, direction correction and straight lines feeding and so on during shield construction process. There is a built-in displacement sensor and a pressure sensor being installed in a certain cylinder to feedback real-time shield tunneling parameters under actual working condition. The proportional valve is controlled by the deviation between feedback signal and a given signal to meet the requirements of online continuously adjustable pressure and speed.

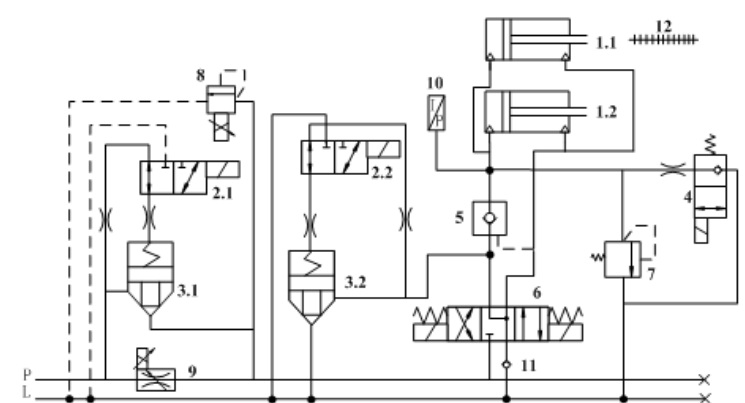

FIGURE I. A SINGLE SET OF HYDRAULIC THRUST SYSTEM SCHEMATIC DIAGRAM

Figure I is the hydraulic thrust system schematic diagram of a shield cylinder. When shield machine is in normal thrust progress, cartridge valves remain closed. Pressure oil enters into the thrust cylinder through the flow speed regulating valve 9. Piston rod tops tightly shield tunnel segment to force cutter head to keep forward. Proportional relief valve 8 can adjust the thrust pressure online on the basis of the deviation between external load from the pressure sensor 10 and pressure command signal. The opening of the throttle valve is changed by the deviation signal between feedback signal from displacement sensor 12 and input command signal. It can change the flow of system so as to realize the closed loop control of thrust speed. When quickly back, solenoid valve 2.1 and 2.2 shift to left and the electromagnetic directional valve 6 located on the left side. Proportional speed regulating valve 9 is 
short-circuited by cartridge valve 3.1. Now the system is in the progress of large flow supply to realize fast back of cylinders.

\section{PID CONTROL OF THRUST SYSTEM BASED ON BP NEURAL NETWORK}

According to the analysis of thrust system, the coordination and synchronization movements of hydraulic cylinders in each zone relate to the precision of shield actual tunneling. Improving the pressure and flow controller is the main method to optimize the control performance of the system [3]. When shield construction environment suddenly changes, PID controller cannot be adjusted in real-time according to actual working conditions, so the dynamic performance and stability of the attitude control become poor.

In order to solve the problems above, nonlinear function approximation ability of BP network is used to optimize the conventional PID controller[4]. Through the network learning and training repetitively, control parameters which conform to the current environment are searched out quickly. When the external environment changes, the BP network is able to go on searching quickly. Since the control strategy has the advantage of adjustment on-line of $k_{p}, k_{i}, k_{d}$, especially, it is suitable for the complex shield control system of time-varying nonlinear load and hysteresis under the actual working condition. PID control structure of speed and pressure of thrust system based on BP networks is shown in Figure II.

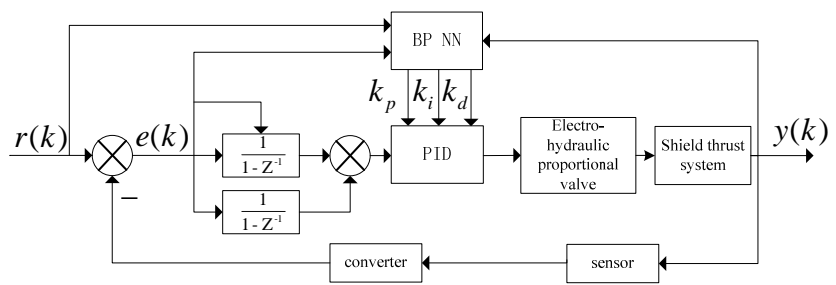

FIGURE II. PID CONTROLLER BASED ON BP NETWORKS

According to the thrust system of shield machine, 3-5-3 type BP neural network model is set up. And then we add the PID control algorithm to the BP network model. Through the network learning and training repetitively, the controller outputs ideal control parameters.

As shown in Figure III, three parameters are taken as the model inputs, there are, the set value $r(k)$ of shield machine speed or pressure, the actual value $\mathrm{y}(\mathrm{k})$ of the thrust speed or pressure and the deviation e $(\mathrm{k})$ between them.

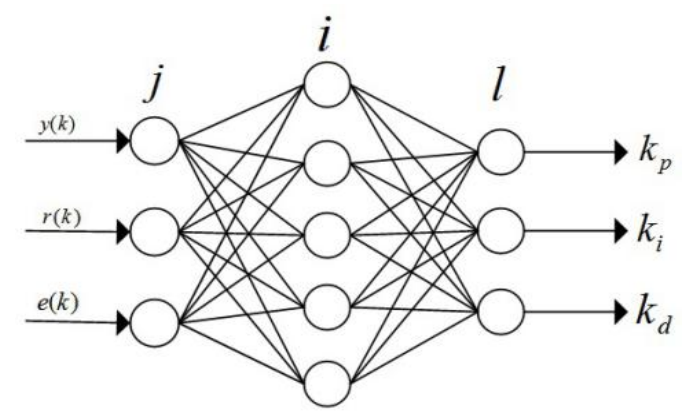

FIGURE III. PID STRUCTURE OF BP NETWORKS
The input and output of BP networks situated in the second layer are as follows:

$$
\begin{aligned}
& H_{i}^{(2)}(k)=\sum_{j=0}^{M} w_{i j}^{(2)} X_{j}^{(1)} \\
& i=1,2, \ldots, 5 ; j=1,2, \ldots, 5 \\
& N_{i}^{(2)}(k)=f\left(H_{i}^{(2)}(k)\right) \\
& i=1,2, \ldots ., 5
\end{aligned}
$$

Where, $H_{i}^{(2)}$ is the input of neurons in hidden layer, $w_{i j}$ is the strength of the connection between the first two layers of neurons, $X_{j}$ is the output of the input layer nodes, $N_{\mathrm{i}}^{(2)}$ is the output of a certain node of a hidden layer.

The excitation function of network 2nd layer is symmetric sigmoid function.

$$
f(x)=\frac{e^{x}-e^{-x}}{e^{x}+e^{-x}}
$$

The input and output situated in network 3rd layer are as follows:

$$
\begin{gathered}
H_{l}^{(3)}(k)=\sum_{i=1}^{5} w_{l i} N_{i}^{(2)} \\
l=1,2,3 \\
N_{l}^{(3)}(k)=g\left(H_{l}^{(3)}(k)\right) \\
l=1,2,3 \\
N_{1}^{(3)}(k)=k_{p} \\
N_{2}^{(3)}(k)=k_{i} \\
N_{3}^{(3)}(k)=k_{d}
\end{gathered}
$$

Where, $H_{l}^{(3)}$ is the input of the third layer, $w_{l i}$ is the strength of the connection between the second and the third layer nodes, $N_{l}^{(3)}$ is the output of the third layer neurons.

The output of the hidden layer is $k_{p}, k_{i}, k_{d}$, so the activation function of the layer is nonnegative sigmoid function

$$
g(x)=\frac{1}{2}(1+\tanh (x))=\frac{e^{x}}{e^{x}+e^{-x}}
$$

Performance index function is

$$
E(k)=\frac{1}{2}(r(k)-y(k))^{2}
$$


Where, $E(k)$ is indicator function, $r(k)$ is the sampling input signal, $\mathrm{y}(\mathrm{k})$ is the sampling output signal.

The learning algorithm of weights takes gradient descent algorithm with additional momentum, which solves the problem of slow convergence effectively. The modified algorithm of weights in network third layer is as follows:

$$
\Delta w_{l i}^{(3)}(k)=\alpha \Delta w_{l i}^{(3)}(k-1)+\eta \delta_{l}^{(3)} N_{i}^{(2)}(k)
$$

Where: $\delta_{i}^{(3)}=e(k) \operatorname{sgn}\left(\frac{\partial y(k)}{\partial \Delta u(k)}\right) \frac{\partial \Delta u(k)}{\partial N_{l}^{(3)}(k)} g^{\prime}\left(H_{l}^{(3)}(k)\right)$

$$
g^{\prime}(x)=g(x)(1-g(x))
$$

$$
\Delta w_{i j}^{(2)}(k)=\alpha \Delta w_{i j}^{(2)}(k-1)+\eta \delta_{i}^{(2)} X_{j}^{(1)}(k)
$$

where:

$$
\begin{gathered}
\delta_{i}^{(2)}=f^{\prime}\left(H_{i}^{(2)}(k)\right) \sum_{l=1}^{3} \delta_{l}^{(3)} w_{l i}^{(3)}(k) f^{\prime}(x)=\left(1-f^{2}(x)\right) / 2 \\
\alpha \text { :inertia coefficient, }{ }^{\eta} \text { :learning rate } \\
\text { IV. SySTEM SimULATION }
\end{gathered}
$$

The structure model for the PID controller based on BP networks is established by Simulink. The input ports of the controller are the pressure and flow set value $r(k)$ of thrust system and the actual value $\mathrm{y}(\mathrm{k})$ respectively. By the online calculation for PID controller, we obtain control rule $\mathrm{u}(\mathrm{k})$ and controller parameters on the output end, as shown in Figure IV.

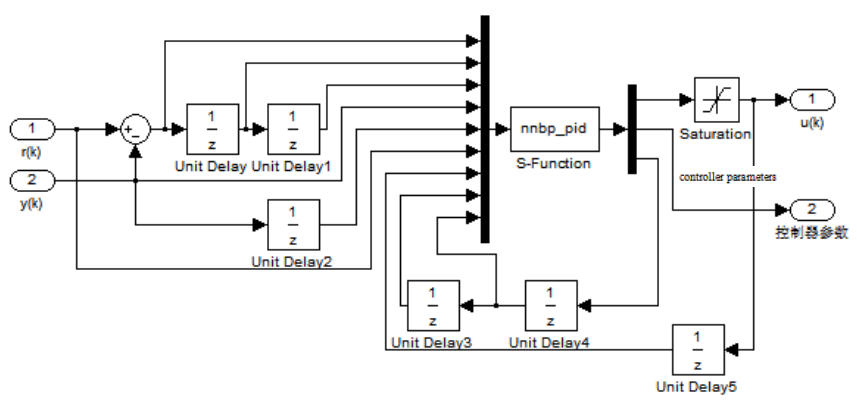

FIGURE IV. THE SIMULATION STRUCTURE OF PID CONTROLLER BASED ON BP NETWORKS

The physical model of thrust system is established by HCD that comes with AMESim software. As shown in Figure V and Figure VI, we realize joint simulation by the interface module of Simulink.

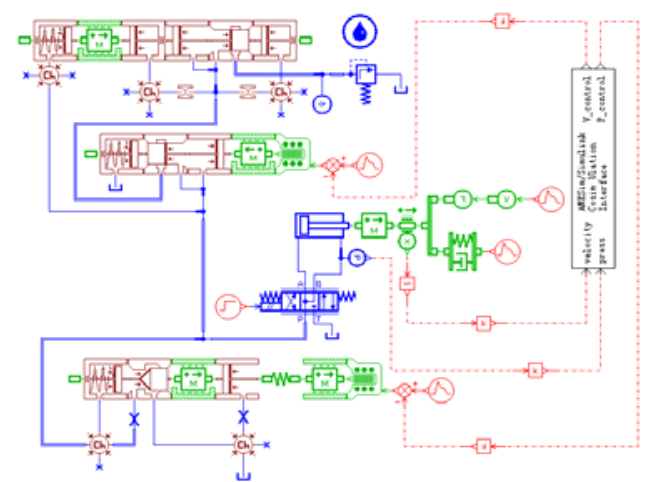

FIGURE V. AMESIM MODEL OF JOINT SIMULATION OF THRUST SYSTEM

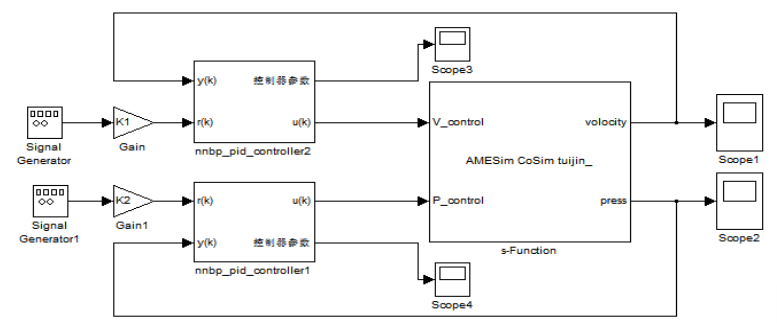

FIGURE VI. THE JOINT SIMULATION OF THRUST SYSTEM

\section{RESUlt ANALYSIS}

\section{A. The Flow Changes While The Load Is Constant}

The propulsive load is $600 \mathrm{KN}$, the flow of the speed regulating valve is $1.2 \mathrm{~L} / \mathrm{min}$ in $0 \sim 5 \mathrm{~s}$ and then $1.8 \mathrm{~L} / \mathrm{min}$ in $5 \sim 10 \mathrm{~s}$. The parameters setting of BP networks are: the efficiency of the network learning is set as 0.20 , the inertia coefficient is 0.05 , the simulation time is $10 \mathrm{~s}$, the sampling time is $0.02 \mathrm{~s}$. When other parameters setting are complete, we begin to carry out joint simulation for thrust system. Figure VII and figure 8 are the simulation curves of thrust speed and pressure when we use the conventional PID controller and the PID controller based on BP neural network respectively.

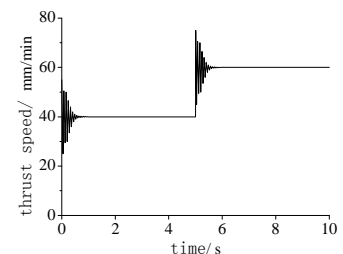

a) speed curve

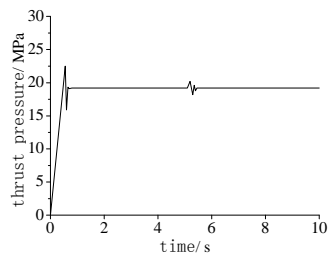

b) pressure curve
FIGURE VII. THE SPEED AND PRESSURE CURVES FOR CONVENTIONAL PID CONTROLLER

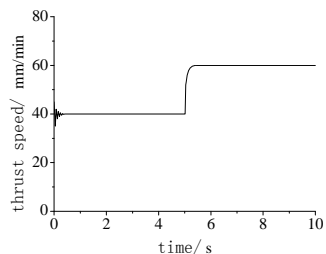

a) speed curve b) pressure curve

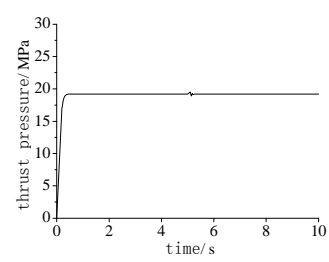

FIGURE VIII. THE SPEED AND PRESSURE CURVES FOR PID CONTROLLER BASED ON BP NETWORK 
As the response curves in Figure VII and Figure VIII shown, the thrust speed is from $40 \mathrm{~mm} / \mathrm{min}$ to $60 \mathrm{~mm} / \mathrm{min}$ rapidly after a reasonable range of oscillation because of flow mutation of the hydraulic cylinder. The system pressure appears fluctuates in small scope near 19.20 MPa. When we use the PID control strategies based on BP neural network, the thrust speed reaches steady state after a short time of adjustment and the phenomenon of non-overshoot exists. The response time can be improved to about $0.4 \mathrm{~s}$ effectively. Maximum amplitude reduces to a half and a sudden change of flow quantity has little effect on pressure. Thus this control method has good trace-ability and adaptive ability.

\section{B. The Load Changes While The Flow Is Constant}

Within the first five seconds, the load is set at $600 \mathrm{KN}$ and within the later five seconds, the load is set at $800 \mathrm{KN}$. The flow of the speed control valve is set at $1.2 \mathrm{~L} / \mathrm{min}$. Then the simulation curve of thrust speed and pressure is as shown in Figure IX and Figure X.

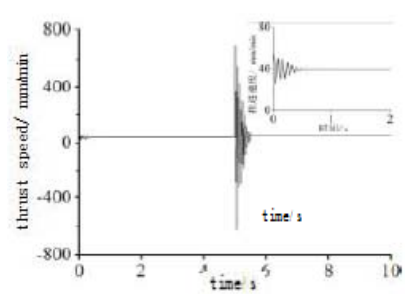

a) speed curve

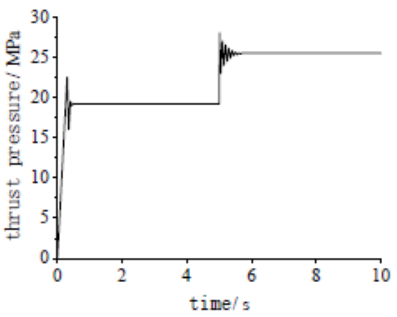

b) pressure curve
FIGURE IX. THE SPEED AND PRESSURE CURVES FOR CONVENTIONAL PID CONTROLLER

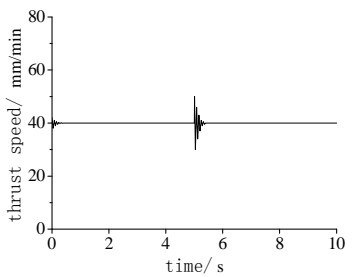

a)speed curve

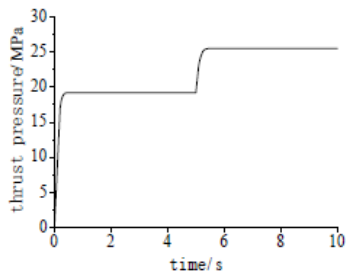

b)pressure curve

FIGURE $X$. THE SPEED AND PRESSURE CURVES FOR PID CONTROLLER BASED ON BP NETWORKS

With the PID control strategies, as the simulation curve in figure 9 shown, the system pressure increases from 19.20 MPa to $25.48 \mathrm{MPa}$ rapidly within $1.2 \mathrm{~s}$ after a small fluctuation when the thrust load changes suddenly. But the maximum value of speed reaches about $600 \mathrm{~mm} / \mathrm{min}$ for a moment when it is at $5 \mathrm{~s}$. The system appears serious overshoot and the control performance of speed becomes poor. The speed curve range in figure 9(a) chooses -800 to 800 in order to display the variation range of the curve easily. The reason of the phenomenon is that the sudden change of thrust load will lead to the abrupt change of hydraulic cylinder flow. This has a great influence on the speed. The conventional PID controller can not meet the requirements of the speed control precision accordingly. With the PID control strategies based on BP neural networks, the speed amplitude reduces to about $50 \mathrm{~mm} / \mathrm{min}$ as shown in figure 10 . The amplitude returns to the original steady state value at $40 \mathrm{~mm} / \mathrm{min}$ quickly after $0.4 \mathrm{~s}$. The abrupt change of load has a weaker influence on system speed, and therefor the PID controller based on BP networks is more suitable for the time-varying system causing by external load and interference factors. It has excellent robustness.

\section{CONCLUSION}

The PID controller based on BP neural network is designed for the thrust hydraulic control system with time-varying load in this paper. The controller can adjust control parameters online. The control precision of thrust pressure and speed and the adaptability, stability of system are improved. The simulation results show that the control method can effectively weaken the disturbance of the various uncertain factors such as model perturbation and the change of the geological condition in shield tunnel construction, to provide a theory reference for the design of thrust hydraulic control system of shield machine in the complicated formations.

\section{ACKNOWLEDGMENT}

This study is supported by the open project of State Key Laboratory of Shield Machine and Boring Technology(201403).

\section{REFERENCES}

[1] Gong Guofang, Hu Guoliang. Control analysis of thrust hydraulic system for shield tunneling machine[J]. China Mechanical Engineering,2007,12(18) :1391-1395.

[2] Liu Guobin, Gong Guofang, Zhu Beidou. Adaptive PID control for thrust speed of the shield based on BP neural networks[J]. Journal of Engineering Design, 2010, 17(6): 454-458.

[3] $\mathrm{Hu}$ Guoliang, Gong Guofang, Yang Huayong. Pressure and flow compound control analysis of thrust hydraulic system for shield tunneling machine[J].Journal of the China Coal Society, 2006, 1(31): 125-128.

[4] Shi Hu, Yang Huayong. Adaptive PID control for thrust speed of shield based on single neuron[J]. China Mechanical Engineering, 2009, 20(2):38-41. 